\title{
FACTORS OF THE USE OF LEARNING ANALYTICS DASHBOARD THAT AFFECT METACOGNITION
}

\author{
Li Chen ${ }^{1}$, Min Lu², Yoshiko Goda ${ }^{3}$, Atsushi Shimada ${ }^{4}$ and Masanori Yamada ${ }^{2}$ \\ ${ }^{1}$ Graduate School of Human-Environment Studies, Kyushu University ${ }^{1}, 744$, Motooka, Nishi-ku, \\ Fukuoka 819-0395, Japan \\ ${ }^{2}$ Faculty of Arts and Science, Kyushu University, 744, Motooka, Nishi-ku, Fukuoka 819-0395, Japan \\ ${ }^{3}$ Research Center for Instructional Systems, Kumamoto University, 2-39-1, Kurokami, Chuo-ku, \\ Kumamoto, 8600862 Japan \\ ${ }^{4}$ Faculty of Information Science and Electrical Engineering, 744, Motooka, Nishi-ku, Fukuoka 819-0395, Japan
}

\begin{abstract}
In this study, we used a learning analytics dashboard (LAD) in a higher education course to support students' metacognition and evaluated the effects of its use. The LAD displays students' reading path and specific behaviors when viewing digital learning materials. The study was conducted on 53 university students to identify the factors that affected metacognition changes in terms of their awareness and behavior dimensions when using the LAD. In terms of results, first, the students' perception of visual attraction for the LAD, and behaviors related to reflection such as deleting annotations they had previously added, positively affected the changes in the knowledge of cognition dimension of metacognition. Second, students' perception of behavioral changes by using the LAD had positive effects on the regulation of cognition dimension of metacognition. However, the behaviors of using some cognitive tools, negatively affected knowledge of cognition, which indicated the necessity to provide more guidance or feedback to students.
\end{abstract}

\section{KEYWORDS}

Metacognition, Self-Regulated Learning, Learning Analytics Dashboard, Learning Behaviors

\section{INTRODUCTION}

Self-regulated learning (SRL) is a self-directive process that is important for learners to gain academic achievements such as good grades and academic skills (Zimmerman, 1998, 2002). In SRL, which includes the metacognition and motivation dimension, learners apply their cognitive learning strategies when conducting specific tasks, during which time they also monitor and reflect on their own learning processes (Tobias \& Everson, 2009). Metacognition, the self-perception of the cognitive learning process, is an important dimension of SRL in which it is essential for learners to make sense of their own learning processes and regulate their learning effectively and efficiently (Tobias \& Everson, 2009).

In order to support learners' metacognition, visualization is a useful tool to make learners aware of their learning processes and learning behaviors by providing specific information regarding their learning activities (Yen et al., 2018). As technology advances, various types of data can be collected through a learning analytics (LA) approach, including learning log data to represent learning behaviors. In SRL, the LA approach is useful for recording and collecting learners' learning processes and informing learners and instructors in a salient way such as with the dashboard. In this paper, we used a learning analytics dashboard (LAD) to support students' metacognition and examined the effects of the use of LAD on awareness and behavior dimensions on metacognition changes; this is useful to identify the factors that should be considered in the design of the use of the LAD. 


\section{LEARNING ANALYTICS APPROACHES FOR METACOGNITION}

Metacognition includes two dimensions: knowledge about cognition and self-regulation of cognition (e.g., Hofer et al., 1998; Jacobs \& Paris, 1987). The Knowledge about cognition dimension concerns learners' awareness and perceptions of their cognitive processes, which generally includes declarative knowledge, procedural knowledge, and conditional knowledge (Jacobs \& Paris, 1987). Self-regulation of cognition, which refers to the plan and control of these cognitive processes, generally includes three metacognitive phases: planning, monitoring, and regulating (Hofer et al., 1998). When learners engage in metacognition-related activities during their learning, they usually need to use relevant knowledge and awareness about how to conduct cognitive processes, as well as plan, control, and monitor these processes.

However, these cognition and metacognition-related processes are complex and include several learning strategies; this causes difficulties in measuring metacognition (Zimmerman, 2002). As one of the most widely used metacognition measurements, 'self-reported inventory' showed some limitations regarding validity and accuracy. For example, it is difficult for learners to remember all their behaviors during the learning process, which would influence their answers during measurement (Tobias \& Everson, 2009). Therefore, in addition to self-reporting inventory, other metacognition measurements capable of recording learners' learning processes are expected. The LA approach has demonstrated great potential in collecting and recording learning log data from the whole learning processes in a continuous and automatic way, without the interference of learners' perceptions or the external environment (Shimada et al., 2015; Yamada et al., 2016; Yin et al., 2015). As such, this can effectively minimize the limitations of self-reported inventory.

However, effectively providing and displaying learning information to learners and instructors remains a challenge. LAD is the integration of the LA approach with the concept of a dashboard, which visualizes the specific information regarding learning data, learning patterns, and behaviors in a concise, direct way (Teasley, 2017). LAD can help learners monitor and reflect on their learning processes, particularly in terms of recognizing what they have been doing and what they should be doing based on their learning goals (Klerkx et al., 2017).

Previous research has focused on the design and development of LAD and the effects of its use in educational environments, aiming to improve instructors' teaching and learners' learning. For example, in higher education, student-facing LADs have often been used to support online learning (e.g., Bodily et al., 2018; Kim et al., 2016) by visualizing students' operations on LADs during the online course. As a result, the positive effects of LADs on final scores (Kim et al., 2016), and the positive perceptions of LAD, were confirmed (Bodily et al., 2018). However, in spite of the findings, both of these studies indicated a necessity to motivate students to use LADs consistently. In this regard, it is necessary to take students' expectations into consideration and provide them with a personalized dashboard, statistical data information, feedback, and advice on how to improve and how to interpret the results (Teasley, 2017).

Based on the insights from previous research, we designed and developed an LAD to support metacognition. The purpose of this study is to explore the effects of LAD on supporting metacognition. Specifically, in order to improve the design and practical use of the LAD, we aim to identify the factors that impact metacognition changes in terms of students' awareness and behavior dimensions while using the LAD.

\section{METHODS}

\subsection{Design Overview}

This study was conducted in an education course where 79 undergraduate students participated. The course was conducted over a 7-week period, with one 90 minute lecture per week. During the course, students studied some basic topics regarding the challenges of modern education and learning, as well as a theoretical background in education. Orientation, which aimed to help students become familiar with the theme and the procedure of the course, was held in the first week. In Week 2 Week 8, before each lecture, the teacher distributed advanced learning materials on BookRoll, which was used as an e-book reader system. Students were asked to review the learning materials before lectures and access the learning materials during lectures. 
Meanwhile, students were provided another system, the LAD named "Metaboard," to support their metacognition. On the Metaboard, students could see their own information and the class average of BookRoll learning behaviors, in an attempt to help them contextualize their learning situation relative to the class average.

\subsection{BookRoll System and Metaboard}

In this study, two systems were used throughout the course. First, the BookRoll system was used as an e-book reader system to store and distribute digital lecture materials. Students read this material by using functional tools such as highlighting contents, adding annotations, and posting bookmarks within the materials (Ogata et al., 2017). All the operations of these functional tools were recorded and collected as learning logs to represent students' learning behaviors in terms of reading and learning the material.

Second, an LAD named Metaboard was used to support students' metacognition. The Metaboard was designed to display details about how students read digital lecture materials on BookRoll (Chen et al., 2019a). The Metaboard consisted of two parts, a reading path overview and a detailed view. In the reading path overview (Figure 1), students could view their own score (left) and the class average (right) in the reading path of the lecture materials, including the time they spent reading each page and whether markers (highlights) or memos (annotations) were used on each page. All the information was divided into in-class and out-of-class view. The meaning of each element is as follows (Lu et al., 2020a, b):

- The node represents the page number, and the color intensity of a node indicates the reading duration for that page (the darker the color, the more time the learners spent reading).

- The thickness of the link indicates the frequency of viewing the page (the thicker the link, the more learners read on that same path).

- The color of the links indicates the direction of the reading path (light gray: turn to the next page; dark gray: turn to the previous page; mint: jump forward; orange: jump backward).

- The smaller circles (circles with the letter $\mathrm{H}$ and $\mathrm{M}$ ) indicate highlight markers $(\mathrm{H})$ or memo annotations (M) included on that page, and the color intensity indicates the total number of tools used.

When students click a page node, a detailed view of this page is provided (Figure 2), including the specific numbers of highlight markers and memo annotations, as well as the reading time for that page.

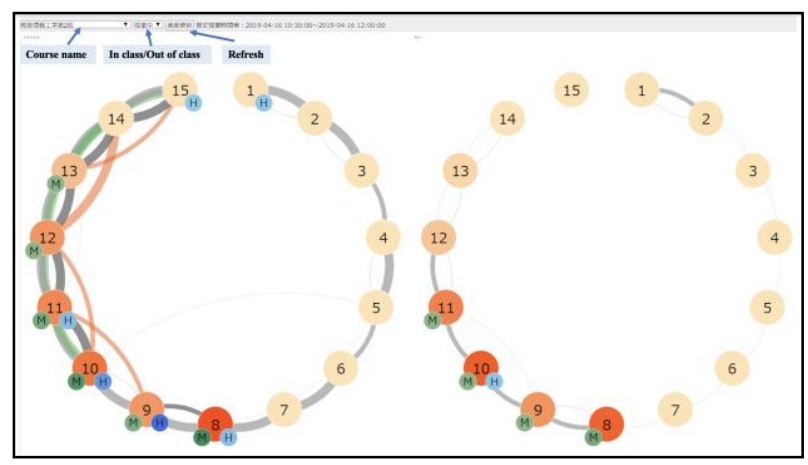

Figure 1. Reading path overview of Metaboard

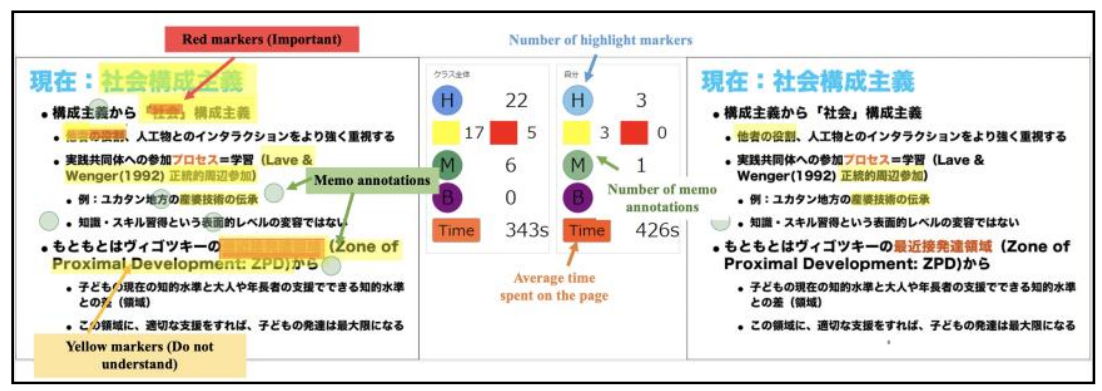

Figure 2. Detailed view of Metaboard 


\subsection{Data Collection and Analysis}

Data was collected using two methods: questionnaires and learning logs. Before and after the whole course, students were asked to answer the Metacognitive Awareness Inventory (MAI) (Harrison \& Vallin, 2018) to assess any changes in their awareness of metacognition. After the course, students completed the LAD success questionnaire (Park \& Jo, 2019) to evaluate the effectiveness of the Metaboard. Both MAI and LAD success questionnaire were translated into Japanese and the items were revised minorly to suit Japanese university students. In addition, all learning logs from BookRoll and Metaboard were collected. Although 79 students initially enrolled in this course, due to absences and missing data from the questionnaires, 53 students' data were collected and analyzed for this study.

\subsubsection{Metacognitive Awareness Inventory (MAI)}

As presented in Table 1, the pre-post MAI consists of two dimensions: knowledge of cognition (what cognitive knowledge students are using) and regulation of cognition (how students regulate their learning by using cognitive and metacognitive strategies). The dimensions contained a total of 19 items.

Table 1. The descriptions of pre-post MAI

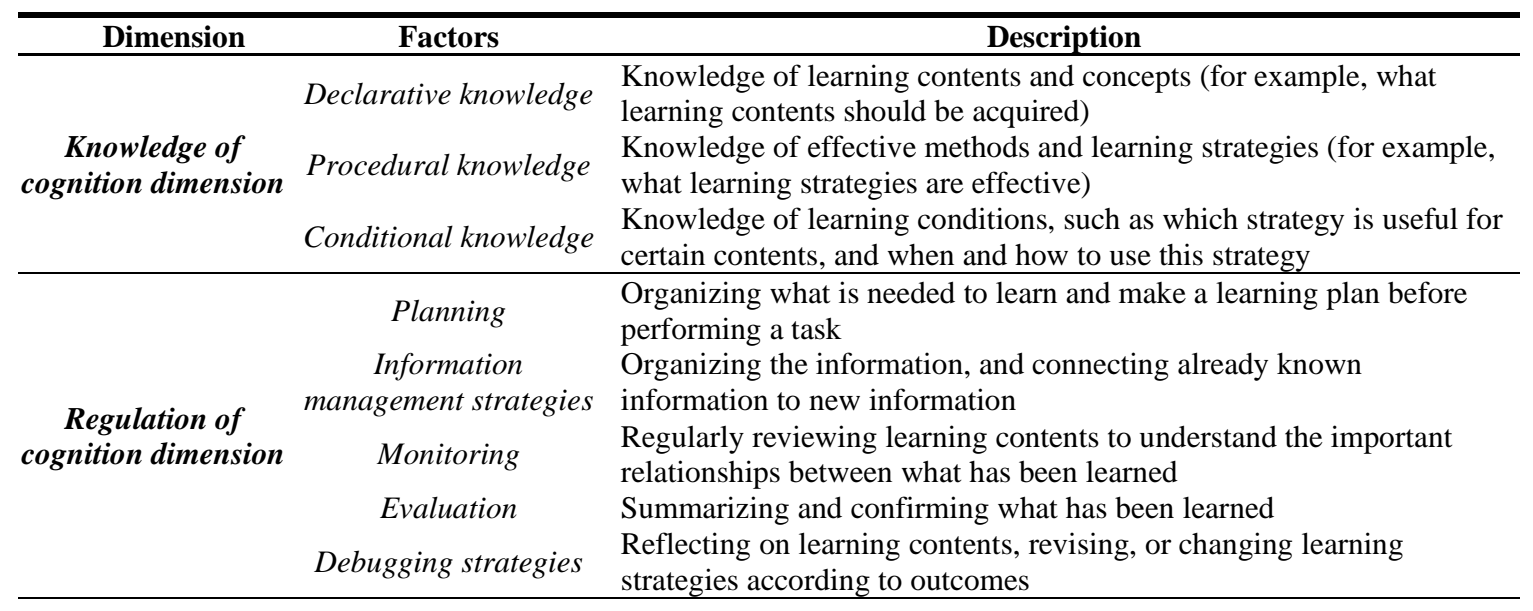

\subsubsection{LAD Success Questionnaire}

As Table 2 shows, the LAD success questionnaire consisted of five dimensions: visual attraction, usability, understanding level, perceived usefulness, and behavioral changes, and contained a total of 28 items.

Table 2. The descriptions of the post-LAD success questionnaire

\begin{tabular}{|c|c|}
\hline Dimension & Description \\
\hline Visual attraction & $\begin{array}{l}\text { Conciseness of the LAD's visual elements (e.g., graphs), the way information was } \\
\text { presented, and the appropriate amount of presented information }\end{array}$ \\
\hline Usability & Ease of access and use of the LAD \\
\hline Understanding level & Degree of understanding of the information presented by the LAD \\
\hline Perceived usefulness & Degree of usefulness of the information presented in the LAD for learning activities \\
\hline Behavioral changes & $\begin{array}{l}\text { Tendency to change learning behaviors such as improving learning motivation, setting } \\
\text { learning plans, and managing learning activities through the use of the LAD }\end{array}$ \\
\hline
\end{tabular}

\subsubsection{Learning logs of BookRoll System and Metaboard}

Table 3 presents descriptions of the learning logs, which recorded the students' operations on BookRoll (ten types) and Metaboard (two types). The learning logs on the BookRoll system were collected and analyzed because Metaboard visually presented the data from BookRoll. 
Table 3. The descriptions of learning logs

\begin{tabular}{ccl}
\hline System & Learning logs & \\
\hline Next & Turn to next page \\
Prev & Turn to previous page \\
Add_Ant & Add an annotation \\
Del_Ant & Delete an annotation \\
Add_Bm & Add bookmarks to learning material \\
& Del_Bm & Delete a bookmark \\
BookRoll system & Add_red_Mk & Add markers (highlight text) within a page in red when students think the \\
& Del_red_Mk & text is important \\
& Delete red markers \\
& Add_yel_Mk & Add markers (highlight text) within a page in yellow when students do not \\
& Del_yel_Mk & understand the text \\
& Delete yellow markers \\
\hline \multirow{2}{*}{ Metaboard } & Open_tool & Access to Metaboard \\
& Click the page icon to view the specific information of each page slide \\
\hline
\end{tabular}

\subsubsection{Variables and Data Analysis}

In order to explore the factors that affect changes in students' metacognition in terms of the awareness and behavior dimensions of using the LAD, a stepwise multiple regression analysis was conducted. The abovementioned ten types of learning behaviors on BookRoll and two types of learning behaviors on Metaboard, as well as the results of the post-LAD success questionnaire, were used as independent variables to predict the two dependent variables of metacognition changes: the changes in knowledge of cognition and regulation of cognition in the MAI. Regarding multicollinearity, the Variance Inflation Factor (VIF) values were checked. The VIF values ranged from 1.00 to 2.65 , indicating no problems in terms of multicollinearity.

\section{RESULTS AND DISCUSSION}

\subsection{Descriptive Data of Dependent and Independent Variables}

Table 4 presents the descriptive data of the two dependent variables (DVs) (i.e., the difference in knowledge of cognition and regulation of cognition of the pre-post MAI) and independent variables (IVs), which are the sum of the scores from the five dimensions of the post-LAD success questionnaire, ten learning behaviors on BookRoll system, and two learning behaviors on Metaboard.

Table 4. Descriptive data of dependent and independent variables $(\mathrm{N}=53)$

\begin{tabular}{cccccc}
\hline Data collection method & Variable & Average & SD & Minimum & Maximum \\
\hline Pre-post MAI & Difference in knowledge of cognition & -0.72 & 3.03 & -9 & 8 \\
(DVs) & Difference in regulation of cognition & 0.04 & 4.85 & -15 & 10 \\
& Visual attraction & 29.26 & 3.80 & 20 & 35 \\
Post-LAD success & Usability & 15.62 & 2.40 & 10 & 20 \\
questionnaire & Understanding level & 15.87 & 2.93 & 8 & 20 \\
(IVs) & Perceived usefulness & 15.81 & 2.66 & 10 & 20 \\
& Behavioral changes & 35.62 & 5.65 & 22 & 45 \\
& Next & 1285.92 & 797.55 & 177 & 3546 \\
& Prev & 475.34 & 364.72 & 26 & 1517 \\
Learning logs on & Add_Ant & 12.13 & 24.71 & 0 & 132 \\
BookRoll system & Del_Ant & 0.55 & 1.15 & 0 & 6 \\
(IVs) & Add_Bm & 0.58 & 1.34 & 0 & 6 \\
& Del_Bm & 0.36 & 0.88 & 0 & 4 \\
& Add_red_Mk & 75.96 & 87.6 & 0 & 438 \\
& Del_red_Mk & 6.75 & 12.86 & 0 & 59 \\
& Add_yel_Mk & 33.3 & 36.54 & 0 & 160 \\
\hline Learning logs on & Del_yel_Mk & 3.42 & 5.99 & 0 & 28 \\
Metaboard (IVs) & Open_Mb & 4.17 & 2.78 & 0 & 16 \\
\hline
\end{tabular}




\subsection{Effects of the use of BookRoll and Metaboard on Metacognition Changes}

\subsubsection{Effects on the Changes in Knowledge of Cognition}

As presented in Table 5, when the difference in knowledge of cognition was used as the dependent variable, the independent variables Understanding level $(\beta=.300, \mathrm{p}=.017)$ from the post-LAD success questionnaire, and Add_Ant $(\beta=-.535, \mathrm{p}=.006)$, Del_Ant $(\beta=.632, \mathrm{p}=.002)$, Add_Bk $(\beta=-.306, \mathrm{p}=.022)$, Del_yel_Mk $(\beta=-.395, \mathrm{p}=.002)$ from the learning $\operatorname{logs}$ accounted for $28.11 \%$ of test score variance: $\mathrm{F}(5,47)=5.07$, $\mathrm{p}=.001$. The results indicate that students' knowledge of cognition awareness increases in accordance with an increase in visual attraction of the LAD assessment and the deleting of annotations (Del_Ant). However, their knowledge of cognition awareness tends to decrease with an increase in the learning behaviors of adding annotations (Add_Ant), adding bookmarks $\left(A d d \_B m\right)$, and deleting yellow markers (Del_yel_Mk).

The awareness of knowledge of cognition is representative of the cognitive knowledge students use in their learning. According to the results, first, the higher assessment of the visual attraction of Metaboard and the learning behavior of Del_Ant showed positive effects on the change in knowledge of cognition. Visual attraction means that a dashboard contains useful information in a concise, direct, and clear form (Park \& Jo, 2019). In addition to providing appropriate graphical representations, it is also necessary to take users' needs and expectations into consideration (Teasley, 2017). Therefore, we required students to answer free-form questions regarding their perceptions and reflections on the use experience of the Metaboard. According to their answers, some students felt that "the provided information was easy to view in graphs," and they also provided some suggestions to improve the visual effects such as "adding functions to adjust the graphs" or "adjusting the thickness of the lines to make it clearer." This information indicates that many students pay attention to the visual attention of an LAD, and considered the visual elements to have an impact on the effective use of the Metaboard. Another factor, Del_Ant indicates students' deleting previously-added annotations. In previous studies, annotation tools were shown to be positively related to students' participation during a lecture (Chen et al., 2020). Students always deleted annotations after they reconsidered or reflected on the content, which is related to metacognition behaviors. Thus, actively deleting or revising annotations is considered effective in helping students reflect on the contents, and understand what content is important and what strategies should be used, which is the main point of knowledge of cognition.

In contrast, the behaviors of $A d d \_A n t, A d d \_B m$, and $D e l \_y e l \_M k$ had negative effects on the change in knowledge of cognition. Using a cognitive tool such as a bookmark proved to be effective in helping students understand content, and was positively related to the learning performance (Van Horne et al., 2016). However, when using such functional tools to support students' cognitive and metacognitive strategies, it is necessary to provide students with guidance about the use of tools and related strategies (Chen et al., 2019b). The lack of guidance and feedback about the use of annotation tools seemed to be one reason for the negative effects on changes in knowledge of cognition. Del_yel_Mk indicates students deleting yellow markers, which were the parts they did not understand well. This behavior can be related to reflection, which is proven to have a positive relationship with learning performance (Chen et al., 2019b) and is effective during discussion or communication with others (Chen et al., 2020). However, some previous studies also indicate occasions when marker tools were ineffective in terms of learning. When students re-read or reviewed content, it is possible that they ignored non-highlighted text, making it difficult to construct overall knowledge (Van Horne et al., 2016; Yue et al., 2015). Therefore, similarly to the use of the annotation and bookmark tools, some specific and direct marker tool guidance from instructors is essential (Van Horne et al., 2016).

Table 5. Results of the regression analysis predicting difference in knowledge of cognition with LAD success assessment and learning behaviors $(\mathrm{N}=53)$

\begin{tabular}{ccccc}
\hline Variable & $\mathbf{B}$ & $\mathbf{S E} \mathbf{B}$ & $\boldsymbol{\beta}$ & $\mathbf{t}$ \\
\hline Visual attraction & 0.311 & 0.125 & 0.300 & $2.48^{* *}$ \\
Add_Ant & -0.066 & 0.023 & -0.535 & $-2.87^{* *}$ \\
Del_Ant & 1.663 & 0.504 & 0.632 & $3.30^{* *}$ \\
Add_Bm & -0.696 & 0.293 & -0.306 & $-2.37^{*}$ \\
Del_yel_Mk & -0.200 & 0.061 & -0.395 & $-3.30^{* *}$ \\
& & & $R^{2}=.350$, Adjusted $R^{2}=.281,{ }^{* *} \mathrm{p}<.01,{ }^{*} \mathrm{p}<.05$
\end{tabular}




\subsubsection{Effects on the Changes in Regulation of Cognition}

As Table 6 shows, when the difference in regulation of cognition was used as the dependent variable, the independent variable Behavioral changes $(\beta=.393, \mathrm{p}=.004)$ of the post-LAD success questionnaire accounted for $13.76 \%$ of the variance in test scores: $F(1,51)=9.30, p=.004$. The results indicate that students' regulation of cognition awareness increases along with an increase in the behavioral changes.

The regulation of cognition concerns the metacognitive behaviors (such as planning or monitoring) of how to conduct cognitive processes during learning. The behavioral changes are the students' tendencies to change learning behaviors after using the LAD (Park \& Jo, 2019). The learning behaviors in behavioral changes include some metacognitive behaviors such as setting learning plans, as well as managing and regulating learning activities based on monitored results. These results are understandable given that these two dimensions have referred similar metacognitive behaviors.

Table 6. Results of the regression analysis predicting differences in regulation of cognition with LAD success assessment and learning behaviors $(\mathrm{N}=53)$

\begin{tabular}{ccccc}
\hline Variable & B & SE B & $\boldsymbol{\beta}$ & t \\
\hline Behavioral changes & 0.337 & 0.111 & 0.393 & $3.05^{* *}$ \\
\hline & & & $R^{2}=.154$, Adjusted $R^{2}=.138,{ }^{* *} \mathrm{p}<.01$
\end{tabular}

\section{CONCLUSIONS}

In order to support students' metacognition, we developed an LAD named Metaboard to visualize students' reading paths and specific information about learning behaviors while viewing digital learning materials. This formative evaluation was conducted on a university education course with 53 participants, to identify the factors that affect students' metacognition changes regarding their awareness and behavior dimensions by using the LAD. As the results indicate, first, students' perceptions of visual attraction for LAD assessment and learning behaviors were related to reflection; for example, the act of deleting annotations was found to have a positive effect on changes in the knowledge of cognition dimension. Second, the students' perceptions of behavioral changes for LAD had positive effects on the regulation of cognition dimension. This is easy to understand since these behaviors are all related to metacognitive activities such as planning and monitoring. However, the use of some cognitive tools, such as adding annotations and bookmarks, negatively affected knowledge of cognition, which indicates the necessity to provide guidance or feedback for students. One main implication this study provides is that the Metaboard can be used to support students in reflecting on and making sense of their cognitive activities, based on the comparison with class average.

In a future study, we will revise and improve the Metaboard based on these results. The effects on learning performance should also be examined when evaluating its effectiveness in future research. Finally, the sample size of 53 students is relatively small for a multiple regression analysis; in order to increase the statistical power of our findings, a further study should be conducted with a larger sample size.

\section{ACKNOWLEDGEMENT}

This work was supported by a JST AIP, Grant No. JPMJCR19U1, and JSPS KAKENHI Grant Number JP19H01716, JP20H01727, JP20K19939, Japan. 


\section{REFERENCES}

Bodily, R., Ikahihifo, T. K., Mackley, B., \& Graham, C. R., 2018. The design, development, and implementation of student-facing learning analytics dashboards. Journal of Computing in Higher Education, Vol. 30, No. 3, pp. 572-598.

Chen, L. et al, 2019a. Design of learning analytics dashboard supporting metacognition. Proceedings of 16th International Conference Cognition and Exploratory Learning in Digital Age (CELDA 2019), Cagliari, Italy, pp. 175-182.

Chen, L. et al, 2019b. Direction of collaborative problem solving-based STEM learning by learning analytics approach. Research and Practice in Technology Enhanced Learning, Vol. 14, No. 1, pp 1-28.

Chen, L. et al, 2020. Exploring factors that influence collaborative problem solving awareness in science education. Technology, Knowledge and Learning, Vol. 25, pp. 337-366.

Harrison, G. M., \& Vallin, L. M., 2018. Evaluating the metacognitive awareness inventory using empirical factor-structure evidence. Metacognition and Learning, Vol. 13, No. 1, pp. 15-38.

Hofer, B. et al, 1998. Teaching college students to be self-regulated learners. In: Schunk, D. H., and Zimmerman, B. J. (eds.), Self-Regulated Learning: From Teaching to Self-Reflective Practice, Guilford Press, New York, USA.

Jacobs, J. E., \& Paris, S. G., 1987. Children's metacognition about reading: Issues in definition, measurement, and instruction. Educational Psychologist, Vol. 22, No. 3-4, pp. 255-278.

Kim, J. et al, 2016. Effects of Learning Analytics Dashboard: Analyzing the Relations among Dashboard Utilization, Satisfaction, and Learning Achievement, Asia Pacific Education Review, Vol. 17, No. 1, pp. 13-24.

Klerkx, J. et al, 2017. Learning analytics dashboards. In C. Lang, G. Siemens, A. Wise, \& D. Gašević (Eds.), The handbook of learning analytics (pp. 143-150). Society for Learning Analytics Research (SoLAR).

Lu, M. et al, 2020a. Visualizing Studying Activities for a Learning Dashboard Supporting Meta-cognition for Students. In: Streitz N., Konomi S. (eds) Distributed, Ambient and Pervasive Interactions. HCII 2020. Lecture Notes in Computer Science, Vol. 12203. pp. 569-580. Springer, Cham, New York, USA.

$\mathrm{Lu}$, M. et al, 2020b. Development of a learning dashboard prototype supporting meta-cognition for students, Companion Proceedings of the 10th International Conference on Learning Analytics \& Knowledge (LAK 2020). FrankFurt, Germany, pp.104-106.

Ogata, H. et al, 2017. M2B System: A Digital Learning Platform for Traditional Classrooms in University. Practitioner Track Proceedings of the Seventh International Learning Analytics \& Knowledge Conference (LAK 2017). Simon Fraser University, Vancouver, Canada, pp. 155-162.

Park, Y., \& Jo, I.-H., 2019. Factors that affect the success of learning analytics dashboards. Educational Technology Research and Development, Vol. 67, No. 6, pp. 1547-1571.

Shimada, A. et al, 2015. Informal learning behavior analysis using action logs and slide features in e-textbooks, Proceedings of IEEE ICALT 2015, Hangzhou, China, pp.116-117.

Teasley, S. D., 2017. Student Facing Dashboards: One Size Fits All? Technology, Knowledge and Learning, Vol. 22, No. 3, pp. 377-384.

Tobias, S. and Everson, H. T, 2009. The importance of knowing what you know: A knowledge monitoring framework for studying metacognition in education. In: Handbook of Metacognition in Education, Routledge.

Van Horne, S. et al, 2016. The adoption of mark-up tools in an interactive e-text- book reader. Educational Technology Research and Development, Vol. 64, No. 3, pp. 407-433.

Yamada, M. et al, 2016. Learning Analytics in Ubiquitous Learning Environments: Self-Regulated Learning Perspective. Proceedings of International Conference on Computers in Education 2016 (ICCE 2016), Mumbai, India, pp. 306-314.

Yen, M. H. et al, 2018. A Framework for Self-Regulated Digital Learning (SRDL), Journal of Computer Assisted Learning, Vol. 34, No. 5, pp. 580-589.

Yin, C. et al, 2015. Analyzing the Features of Learning Behaviors of Students using e-Books, Proceedings of International Conference on Computers in Education 2015 (ICCE 2015), Hangzhou, China, pp.617-626.

Yue, C. L. et al, 2015. Highlighting and its relation to distributed study and students' metacognitive beliefs. Educational Psychology Review, Vol. 27, pp. 69-78.

Zimmerman, B. J, 1998. Developing self-fulfilling cycles of academic regulation: An analysis of exemplary instructional models. In: Schunk, D. H., and Zimmerman, B. J. (eds.), Self-Regulated Learning: From Teaching to Self-Reflective Practice, Guilford Press, New York, USA.

Zimmerman, B. J., 2002. Becoming a Self-Regulated Learner: An Overview, Theory into Practice, Vol. 41, No. 2, 64-70. 